\title{
El sistema de salud colombiano, reflexiones sobre su influencia en la salud de la población
}

\author{
The Colombian health system: Reflections on \\ its influence on the health of the population \\ O sistema de saúde colombiano, reflexões \\ sobre sua influência na saúde da população
}

\author{
Carlos Alberto Reina Bolaños ${ }^{1}$
}

\begin{abstract}
Recibido: 20 de agosto 2020 • Enviado para modificación: 7 de octubre 2020 • Aceptado: 23 de agosto 2021 Reina Bolaños, C. A. (2021). El sistema de salud colombiano, reflexiones sobre su influencia en la salud de la población. Revista Ocupación Humana, 21(2), 88-98. https://doi.org/10.25214/25907816.911
\end{abstract}

\begin{abstract}
RESUMEN
Los sistemas de salud son una respuesta social que se modifica y adapta según el momento y lugar en la historia; en la medida que las sociedades se complejizan, estos encuentran nuevos desafíos. Conocer este panorama facilita su interpretación. Este artículo presenta una reflexión que parte de reconocer los principales avances y limitaciones del sistema de salud colombiano, para ampliar el panorama frente a sus posibilidades y necesidades de configuración. Se analizan elementos sociopolíticos y económicos de contexto y se examinan los principales indicadores de salud usados para reflejar la manera como los sistemas de salud responden a la protección de la población. El Sistema General de Seguridad Social en Salud, creado a través de la Ley 100 de 1993, nació en el contexto del desmonte de los Estados de bienestar y el fortalecimiento del neoliberalismo. Con este se han logrando avances en el acceso a los servicios de salud de la población en mayores condiciones de pobreza, sin embargo, se continúan presentando brechas e inequidades. La Terapia Ocupacional no es ajena a estas cuestiones, sus acciones deben propender por el cierre de tales brechas e inequidades sociales.
\end{abstract}

\section{PALABRAS CLAVE}

sistemas de salud, Colombia, salud pública

\footnotetext{
1 Terapeuta Ocupacional. Magíster en Salud Pública. Doctorando en Epidemiología, Universidad de Antioquia. Santiago de Cali, Colombia. carlos.reina@udea.edu.coｉD https://orcid.org/0000-0002-6367-9239
} 


\begin{abstract}
Health systems are a social response modified and adapted according to the time and place in history. As societies become more complex, health systems face new challenges. Knowing this overview facilitates its interpretation. This article presents a reflection that starts from recognizing the main improvements and limitations of the Colombian health system to broaden the perspective regarding its configuration possibilities and needs. Elements of the socio-political and economic context are analyzed, and the primary health indicators used to reflect how health systems respond to the protection of the population are examined. The Sistema General de Seguridad Social en Salud (general system for social security in health), created by the Law 100 of 1993, was born in the context of dismantling the welfare states and the strengthening of neoliberalism. The system has enabled progress in the access to health services for the population in more critical poverty conditions. However, gaps and inequities are still present. Occupational Therapy is not alien to these issues; its actions should tend to close such gaps and social inequities.
\end{abstract}

\title{
KEYWORDS
}

health systems, Colombia, public health

\section{RESUMO}

Os sistemas de saúde são uma resposta social que se modifica e se adapta de acordo com o tempo e o lugar na história; À medida que as sociedades se tornam mais complexas, elas encontram novos desafios. Conhecer esse panorama facilita sua interpretação. Este artigo apresenta uma reflexão que parte do reconhecimento dos principais avanços e limitações do sistema de saúde colombiano, a fim de ampliar o panorama sobre suas possibilidades e necessidades de configuração. São analisados os elementos sociopolíticos e econômicos do contexto e examinados os principais indicadores de saúde que refletem a forma como os sistemas de saúde respondem à proteção da população. O Regime Geral de Previdência Social em Saúde, criado pela Lei no 100 de 1993, nasceu no contexto de desmantelamento dos Estados de bem-estar e do fortalecimento do neoliberalismo. Com isso, houve avanços no acesso aos serviços de saúde para a população em maior condição de pobreza, porém, lacunas e iniquidades continuam aparecendo. A Terapia Ocupacional não é alheia a essas questões, suas ações devem tender a fechar tais lacunas e iniquidades sociais.

\section{PALAVRAS-CHAVE}

sistemas de saúde, Colômbia, saúde pública

\section{Introducción}

Los sistemas de salud son la respuesta social a la enfermedad (Frenk, 2016), un fenómeno que acompaña a la humanidad desde el inicio de su historia y que pone en riesgo su continuidad en el mundo. En efecto, la forma en que abordamos la enfermedad cambia conforme se modifica la forma de ver y relacionarnos con 
el mundo, un lugar que ha sido conquistado y adaptado a las necesidades e intereses de nuestra especie. No obstante, a medida que formamos sociedades más complejas, los retos a los que nos enfrentamos también lo son; para responder a ellos hemos creado herramientas de control social con la promesa del bien común (Foucault, 1977), una promesa en la que encontramos tranquilidad para afrontar aquellos problemas cuya solución se escapa a nuestras capacidades como individuos. Por lo tanto, la enfermedad se convierte en un asunto al que podemos responder y controlar como sociedad. Entender las dinámicas en las cuales se construyen los sistemas de salud es un imperativo para su análisis.

Desde el desmonte de los Estados de bienestar en los años 70, los sistemas de salud alrededor del mundo han sufrido transformaciones encaminadas a reducir el papel del Estado, de garante de la salud de la población a regulador de los actores que intervienen en el sistema (Waitzkin y Jasso-Aguilar, 2013). Estos cambios han estado acompañados de una serie de reformas en las políticas sociales y económicas encaminadas a promover la libre competencia y la globalización comercial de las naciones.

Es en este contexto en el que surgió en Colombia la Ley 100 de 1993, a través de la cual se creó el Sistema General de Seguridad Social en Salud. 28 años después, aún es confuso el rumbo que debe seguir para garantizar el derecho a la salud, puesto que, pese a los logros en la prestación y cobertura de los servicios, siguen sin resolverse asuntos esenciales de inequidad social que determinan que los grupos sociales más vulnerables continúen presentando grandes brechas. A esto se suma la inestabilidad financie- ra del sistema de salud, que por múltiples factores no cuenta con los recursos económicos suficientes para su adecuado funcionamiento.

Este artículo presenta una reflexión que parte de reconocer los principales avances y limitaciones del Sistema General de Seguridad Social en Salud, para ampliar el panorama frente a las posibilidades y necesidades en su configuración. Se analizan algunos elementos sociopolíticos y económicos de contexto y se examinan los principales indicadores que reflejan la respuesta del sistema frente a la protección global de la población.

\section{Bases del Sistema General de Seguridad Social en Salud}

El sistema de salud, como componente del Sistema General de Seguridad Social en Salud, es un servicio público esencial obligatorio a cargo del Estado que "será prestado por las entidades públicas o privadas en los términos y condiciones establecidos en la presente ley" (Ley 100/1993, art. 4). La salud, pensada desde la lógica de la prestación de servicios, responde al modelo bacteriológico de la enfermedad y al de abordaje de factores de riesgo, el primero para las enfermedades infecciosas y el segundo para las crónicas (Idrovo et al., 2008).

Esta forma de organización ha fomentado la competencia entre prestadores de servicios de salud y aseguradores. Ubicar a estos actores en el centro del Sistema General de Seguridad Social en Salud, en algunos casos, ha favorecido y aumentado las condiciones de calidad de los prestadores de servicios de salud, cumpliendo la tesis del modelo de pluralismo 
estructurado según la cual el mercado es el agente encargado de proporcionar los incentivos necesarios para el avance y mejoramiento del sistema de salud. Sin embargo, en lugares en los que el mercado es débil y poco lucrativo, como las zonas rurales y los municipios pequeños, estos beneficios no son observables, por el contrario, las brechas parecen ser cada vez más grandes entre estos territorios y los principales centros poblados. Incluso, actores como los hospitales públicos se han visto perjudicados al no lograr adherirse a las dinámicas de mercado impuestas por el modelo (Romero, 2009).

Las tensiones ideológicas tienen sus bases divididas entre el libertarismo, ideología que ubica la libertad como bien y fin supremo que debe ser defendido $y$ garantizado por el Estado mediante la protección de los llamados "derechos naturales", y el utilitarismo, el cual plantea que las decisiones deben tomarse en términos de sus posibles consecuencias y cómo estas pueden brindar utilidad individual y social al menor costo posible. Estas posturas son el fundamento ético de la economía neoclásica, pensamiento económico comúnmente utilizado en el Sistema General de Seguridad Social en Salud. En contraposición a ellas, John Rawls impulsa el neokantianismo en las decisiones políticas mediante la justicia redistributiva, que pretende organizar instituciones justas y legítimas como medio para alcanzar la justicia social (Arias, 2017).

En virtud de lograr un sistema de salud capaz de afrontar estas dificultades no resueltas se oponen dos ideas comunes. La primera toma como base la necesidad de realizar modificaciones que subsanen parte de las dificultades percibidas sin cambiar la esencia del sistema; la segunda propone cambiar el sistema por uno completamente nuevo, con bases centradas en la salud como derecho fundamental desde la equidad y la justicia social.

Dado que los sistemas de salud dependen completamente de los sistemas de gobierno, la consecución de la segunda opción requiere de la transformación del Estado por otro que no responda al modelo neoliberal, como lo expone la teoría crítica en el modelo de determinación social de la salud de Breilh (2013), el cual busca: "Desentrañar las raíces socio-ambientales de los problemas de salud que genera y reproduce la acumulación, para pensar sobre estas con un sentido crítico y para actuar en una línea de emancipación respecto a los procesos malsanos" (p. 974). Esta alternativa requiere de transformaciones que no son posibles en el corto plazo, por el contrario, requerirían de procesos sociales que se mantengan, transformen y fortalezcan con el tiempo.

El Sistema General de Seguridad Social en Salud instaurado por la Ley 100/1993 ha sido continuamente debatido, cuestionado y atacado por el hecho de llevar la salud al contexto del mercado, acorde con la corriente del neoliberalismo de minimización del Estado y fortalecimiento del mercado como ente regulador de los actores involucrados.

Este planteamiento ha llevado a los sistemas de salud, más aún en el caso colombiano, a presentar varias dificultades conocidas como fallas de mercado, en las cuales no se pueden garantizar las condiciones necesarias para la libre competencia. Esto se debe principalmente a tres factores: el primero es la asimetría de información entre compradores y ofertantes, en donde el cliente, en este 
caso el usuario o la usuaria del servicio de salud, no cuenta con las herramientas suficientes para conocer si el tratamiento suministrado justifica el valor monetario cobrado o si debe tomar otra alternativa de menor costo. El segundo es la protección financiera: comúnmente quien asume el costo del tratamiento médico no es quien lo compra, sino la sociedad en su conjunto, por lo cual la autorregulación que pueden tener el comprador y el ofertante se ve reducida al no asumir los costos de forma directa. El tercer factor es la demanda inducida por los prescriptores, en donde los actores involucrados pueden hacer uso de incentivos perversos para aumentar la demanda de su producto o servicio (Sloan, 2001).

Cuando estas fallas de mercado no pueden ser corregidas por el mercado mismo, entra al escenario el Estado como actor encargado de corregirlas por medio de instituciones políticas y acuerdos sociales. Sin embargo, si el Estado también presenta fallas, siendo la corrupción una grave falla, no logra cumplir completamente con esta labor.

En esta dinámica de libertad de mercado con regulación estatal ha evolucionado el Sistema General de Seguridad Social en Salud colombiano, trayendo consigo múltiples protestas sociales que buscan que este cambie. Dentro de las principales causas de estas acciones colectivas se encuentran el deterioro de le red pública de prestación de servicios de salud, los acuerdos incumplidos por parte del Gobierno y la precarización de las condiciones laborales de los trabajadores y trabajadoras del sector salud (Echeverry-López y Borrero-Ramírez, 2015); condiciones que no han logrado ser resueltas.
El Estado colombiano ha creado diferentes mecanismos para subsanar las dificultades del sistema y mejorar la calidad en la prestación de los servicios de salud, buscando con esto el avance del modelo de prestación de servicios y la reducción de la presión de los múltiples estamentos sociales. Una de las medidas más fuertes fue la reforma a la salud presentada con la Ley 1122/2007, la cual no obtuvo los resultados esperados al ser considerada por la Corte Constitucional como ineficiente para mejorar las condiciones del sistema; esto, al constatar el aumento en el número de tutelas presentadas desde 1999 para exigir la prestación de servicios de salud que podrían comprometer la vida de pacientes (Echeverry-López \& Borrero-Ramírez, 2015). La Corte emitió la sentencia T-760/2008, que exige al Estado dar cumplimiento a la regulación ya prevista en la norma (Echeverry-López y Borrero-Ramírez, 2015). El Ministerio de Salud, tratando de dar respuesta a esta sentencia de la Corte, promulgó a su vez la Ley 1751/2015, más conocida como Ley Estatutaria en Salud, en donde la salud ya es considerada un derecho fundamental de todas y todos los colombianos que debe ser respetado, protegido y garantizado por el Estado.

\section{Tendencias en indicadores de salud}

Desde su puesta en marcha, la regulación del sistema de salud se ha desarrollado de forma gradual sin tener bases claras de su funcionamiento. Esto ha repercutido en que actores como los aseguradores o la industria farmacéutica se hayan fortalecido a costa de los recursos del sistema, recursos finitos por definición y con una demanda cada vez 
más alta a causa de la transición epidemiológica y demográfica, y el avance en las tecnologías médicas. Actualmente se cuenta con un aparato normativo que vigila los posibles detrimentos de los fondos públicos en salud, como puede ser el caso de la estructuración vertical, organización en la que el asegurador presta los servicios de salud bajo su misma empresa, y el uso de los recursos de salud para fines ajenos al sector.

Pese a la continua promesa sin cumplir por parte del Estado de garantizar la salud como derecho, se perciben avances en puntos importantes para la sociedad. Es el caso de la disminución de las tasas de mortalidad infantil, materna y el aumento en la esperanza de vida de colombianos y colombianas. Estos grupos poblacionales son de especial importancia, pues a través de estos indicadores es posible juzgar el desarrollo de un Estado social de derecho en términos de salud, ya que reflejan problemas estructurales de atención y, por lo tanto, de equidad y justicia social.

En el caso de la mortalidad infantil (figura 1) por cada 1.000 nacidos vivos, en 1993 se presentaban 27 muertes, cifra que se encontraba por debajo de la tendencia mundial de 62 casos. Para el año 2017 se presentaron 12 muertes por cada 1.000 nacidos vivos en el país (Our World in Data, 2020a). Estas cifras, aunque se encuentran por debajo de la media mundial, podrían reducirse en mayor medida. Según el Departamento Administrativo Nacional de Estadística [DANE] (2018), se podría hablar de una meta por debajo de 11 casos por 1.000 nacidos vivos para el 2022.

Figura 1. Tasa de mortalidad infantil en Colombia, 1960-2017

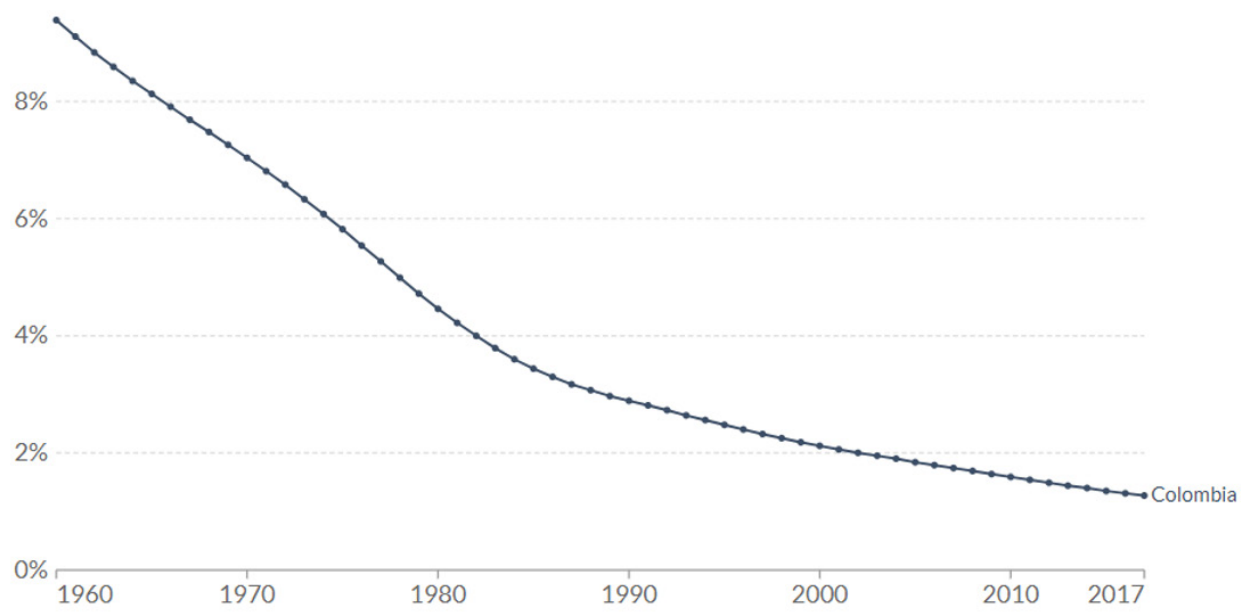

Fuente: Our World in Data (2020a). 
Para el caso de la mortalidad materna, como se puede observar en la figura 2, por cada 1.000 nacidos vivos en 1990 se presentaban 118 muertes maternas, mientras que en el año 2015 esta cifra disminuyó a 64 decesos. En el actual plan de desarrollo colombiano 20182022, Pacto por Colombia, pacto por la equidad (Congreso de Colombia, 2019), la meta de mortalidad materna planteada propone pasar de 51,27 por cada 1.000 nacidos vivos en 2016 a 45 en el 2022, lo cual, según el DANE (2018), corresponde a una meta poco ambiciosa, ya que podría estar por debajo de los 25 casos por cada 1.000 nacidos vivos.

Figura 2. Razón de mortalidad materna en Colombia, 1990-2015

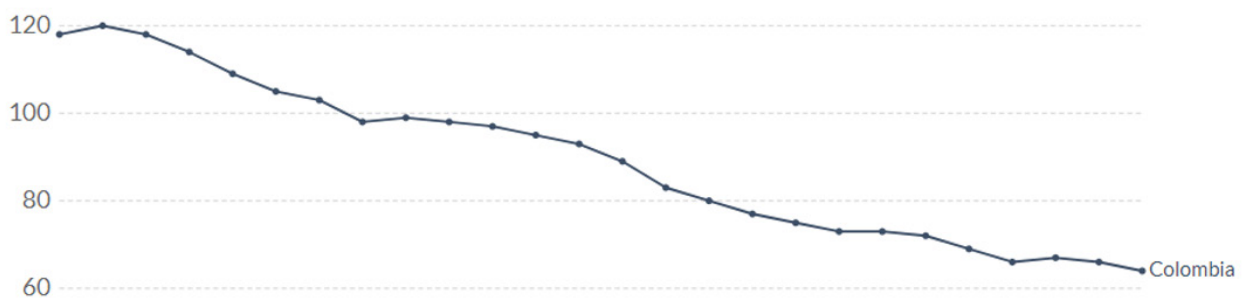

40

20

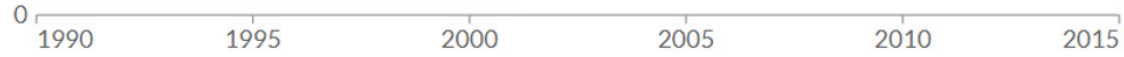

Fuente: Our World in Data (2020b).

La esperanza de vida es un indicador que refleja diferentes condiciones de vida, salud y educación de un país. Entre mejores sean estas condiciones, mayor es su esperanza de vida, por lo cual permite medir el nivel de desarrollo humano de los países (Bilal et al., 2021). Para el caso colombiano, como se observa en la figura 3, en el año 1900 la esperanza de vida al nacer alcanzaba los 29 años de edad, mientras que la media mundial era de 32 años. Para el 2018 esta cifra aumentó a
77 años y en el mundo a 72 (Our World in Data, 2020c)..

\section{Retos estructurales de funcionamiento}

Pese a los avances presentados, surgen nuevas dificultades cada vez más difíciles de controlar. Es el caso de las corporaciones, que actualmente se han 
Figura 3. Esperanza de vida en Colombia, 1900-2018

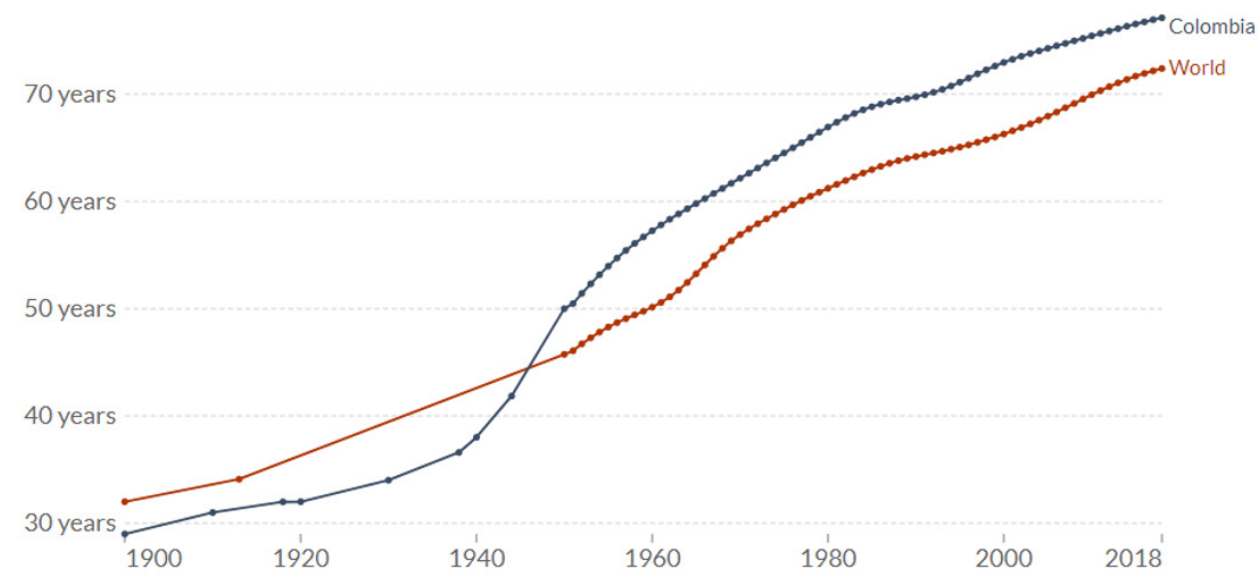

Fuente: Our World in Data (2020c).

convertido en actores influyentes en los ámbitos políticos y económicos, y pueden condicionar el papel del Estado como agente regulador de los sistemas de salud. Esto ocurre mediante el apoyo que dan a grupos políticos que, a su vez, facilitan la obtención de puestos públicos a sus integrantes; una práctica que se conoce como puerta giratoria. De esta forma las corporaciones obtienen ventajas normativas que incrementan su poder en el mercado, creando oligopolios estructurados que pueden, incluso, Ilegar a subordinar el poder del Estado. En el ámbito de la salud, la más fuerte de estas corporaciones es la industria farmacéutica (Jasso-Aguilar y Waitzkin, 2008).

La concentración de poder es un riesgo para el mantenimiento de las metas alcanzadas o para los posibles avances de los sistemas de salud, puesto que confronta tanto los postulados del neoliberalismo como los del Estado social de derecho. Por un lado, las condiciones del mercado favorecen a un grupo reducido de actores que acumulan la mayor parte del capital disponible, con lo cual se afectan el libre mercado y el libre comercio, premisas de esta teoría política económica. Por otra parte, el Estado no cuenta con las capacidades necesarias para regular a estos actores y corregir las fallas del mercado, sin embargo, es a él a quien se acude para garantizar la protección de los derechos sociales por encima de otros intereses.

Los avances del Sistema General de Seguridad Social en Salud han permitido el acceso a los servicios de salud de la población en mayores condiciones de pobreza, con los que anteriormente no contaban. Además de esto, la reducción del gasto de bolsillo es otro de los logros que ha permitido la protección económica en casos de enfermedades de alto costo. Sin embargo, los temas de inequidad y de calidad frente a la prestación de servicios de salud hacen parte de los gran- 
des retos a los cuales solo se ha logrado dar respuesta de forma marginal (Agudelo et al., 2011). Esto expone las limitaciones de un modelo de salud basado en el aseguramiento que actualmente es considerado como universal, pero deja de lado cuestiones constitutivas del sistema, como la necesidad de la reducción del desempleo, puesto que este se pensó sobre la lógica del aumento gradual del régimen contributivo con capacidad de pago frente al subsidiado, comportamiento que no se ha cumplido (Mendieta González y Jaramillo, 2019) .

\section{Reflexiones finales}

Juzgar el estado del sistema de salud colombiano presenta un desafío ético e ideológico, dadas las múltiples interpretaciones e intereses que se tejen a su alrededor. En este debate deben primar la razón y la responsabilidad, debido a que la salud no es el origen de las desigualdades sociales, pero si puede acrecentarlas. La Terapia Ocupacional, como profesión de la salud, no es ajena a estas cuestiones y sus acciones deben propender por el cierre de brechas e inequidades sociales detectadas en el sistema.

Es importante entender que los sistemas de salud deben ir más allá de la prestación de servicios, puesto que estos son solo una parte de lo que es la salud, vista desde el modelo de determinantes sociales (Cárdenas et al., 2017). Esta perspectiva plantea una serie de determinantes estructurales e intermedios que influyen en el estado de salud de la población y que pueden ser abordados en el mediano y el largo plazo. En las circunstancias actuales es posible abordar los determinantes estructurales de posición socioeconómica, clase social, educación, ocupación e ingresos. Si los servicios de salud logran responder a las particularidades de la población en términos de estos factores se podría mejorar el funcionamiento del sistema acercándolo a la población, disminuyendo las barreras y emergiendo de las realidades locales.

Son múltiples los retos que afronta el sistema de salud colombiano, con preguntas para las que aún no tenemos respuesta: ¿cómo aprender a convivir y tomar decisiones colectivas que contemplen a la dignidad humana como principio rector? Y, de conseguirlo, ¿Cómo poder controlarlo? Encomendar una responsabilidad y poder a una organización social requiere de la vigilancia constante del mismo regulador. El compromiso es la primera responsabilidad. Como terapeutas ocupacionales, profesionales de la salud y ciudadanas y ciudadanos colombianos, las posibilidades de acción frente al mejoramiento del sistema de salud están enmarcadas en la participación en la toma de decisiones que se dan en el día a día, desde la calidad con la que se presta un servicio hasta la propuesta económica y de salud por la cual se vota en los diferentes tipos de elecciones. Solo de esta forma se pueden fortalecer las instituciones que sostienen y regulan el sistema y garantizar que estas no pierdan su foco, el cual debe ser, siempre, la salud poblacional. 


\section{Referencias}

Agudelo, C. A., Cardona, J., Ortega, J. y Robledo, R. (2011). Sistema de salud en Colombia: 20 años de logros y problemas. Ciência \& Saúde Coletiva, 16, 2817-2828. https://doi. org/10.1590/S1413-81232011000600020

Arias, S. (2017). Epidemiología, equidad en salud y justicia social. Revista Facultad Nacional de Salud Pública, 35(2), 186196. https://doi.org/10.17533/udea.rfnsp. v35n2a03

Bilal, U., Hessel, P., Perez-Ferrer, C., Michael, Y. L., Alfaro, T., Tenorio-Mucha, J. Friche, A., Pina, M. F. Vives, A., Quick, H., Alazraqui, M., Rodríguez, D., Miranda, J., Diez-Rux, A. y Salurbal group. (2021). Life expectancy and mortality in 363 cities of Latin America. Nature medicine, 27(3), 463470. https://doi.org/10.1038/s41591-020$\underline{01214-4}$

Breilh, J. (2013). La determinación social de la salud como herramienta de transformación hacia una nueva salud pública (salud colectiva). Revista Facultad Nacional de Salud Pública, 31 (sup.1), 13-27. https://revistas.udea.edu.co/index.php/fnsp/article/ view/16637

Cárdenas, E., Juárez, C., Moscoso, R. y Vivas, J. (2017). Determinantes sociales en salud. Esan.

Congreso de la Republica de Colombia (1993, 23 de diciembre). Ley 100 de 1993. Por la cual se crea el sistema de seguridad social integral y se dictan otras disposiciones. Diario Oficial n. ${ }^{\circ}$ 41.148. http://www. secretariasenado.gov.co/senado/basedoc/ ley_0100_1993.html
Congreso de la Republica de Colombia (2007, 9 de enero). Ley 1122 de 2007. Por la cual se hacen algunas modificaciones en el Sistema General de Seguridad Social en Salud y se dictan otras disposiciones. Diario Oficial n. ${ }^{0}$ 46.506. http://www.secretariasenado.gov.co/senado/basedoc/ ley 1122 2007.html

Congreso de la Republica de Colombia (2015, 16 de febrero). Ley Estatutaria 1751 de 2015. Por medio de la cual se regula el derecho fundamental a la salud y se dictan otras disposiciones. Diario Oficial n. ${ }^{\circ}$ 49.427. http://www.secretariasenado.gov. co/senado/basedoc/ley 1751 2015.html

Congreso de Colombia (2019, 25 de mayo). Ley 1955 de 2019. Por el cual se expide el Plan Nacional de Desarrollo 2018-2022: Pacto por Colombia, Pacto por la Equidad. Diario Oficial n. ${ }^{\circ}$ 50.964. http://www. secretariasenado.gov.co/senado/basedoc/ ley 1955 2019.html

Departamento Administrativo Nacional de Estadística [DANE] (2018). Censo nacional de población y vivienda - 2018. DANE. https://www.dane.gov.co/index.php/estadisticas-por-tema/demografia-y-poblacion/ censo-nacional-de-poblacion-y-vivenda-2018/informacion-tecnica

Echeverry-López, M. E. y Borrero-Ramírez, Y. E. (2015). Protestas sociales por la salud en Colombia: la lucha por el derecho fundamental a la salud, 1994-2010. Cadernos de Saúde Pública, 31, 354-364. http://dx. doi.org/10.1590/0102-311X00030714

Foucault, M. (1977). Historia de la medicalización. Educación médica y salud, 11(1), 3-25. 
Frenk, J. (2016). La salud de la población. Hacia una nueva salud pública. Fondo de Cultura Económica.

Idrovo, A. J., Eslava, J. C., Ruiz-Rodríguez, M. y Rodríguez, J. M. (2008). La otra transición epidemiológica: hitos en el desarrollo de la epidemiología de los factores de riesgo en Colombia. Biomédica, 28(4), 480-496. https://doi.org/10.7705/biomedica.v28i4.54

Jasso-Aguilar, R. y Waitzkin, H. (2008). El Estado, las multinacionales y la medicina contemporánea. Palimpestvs, 6, 71-82.

Mendieta, D. y Jaramillo, C. E. (2019). EL sistema general de seguridad social en salud de Colombia. universal, pero ineficiente: a propósito de los veinticinco años de su creación. Revista Latinoamericana de Derecho Social, (29), 201-218. http://dx.doi.org/10.22201/ iij.24487899e.2019.29.13905

Our World in Data (2020a). Infant mortality rate. Global Change Data Lab. https:// ourworldindata.org/grapher/infant-mortality?tab=chart\&time=1960.. latest\&country $=\sim \mathrm{COL}$

Our World in Data (2020b). Maternal Mortality Ratio, 1990 to 2015. Global Change Data Lab. https://ourworldindata.org/grapher/maternal-mortality?tab=chart\&country $=\sim \mathrm{COL}$
Our World in Data (2020c). Life expectancy, 1900 to 2018. Global Change Data Lab. https://ourworldindata.org/grapher/life-expectancy?tab=chart\&time $=1900 . .2018 \&-$ country $=\mathrm{COL} \sim \mathrm{OWID}$ WRL

Romero, R. V. (2009). Los orígenes y desarroIlos de la APS en Colombia. En R. Vega, N. Acosta, P. Mosquera y O. Restrepo, Atención primaria integral de salud. Estrategia para la transformación del sistema de salud y el logro de la equidad en salud (pp. 53-82). Secretaría Distrital de Salud de Bogotá.

Sloan, F. A. (2001). Arrow's concept of the health care consumer: a forty-year retrospective. Journal of Health Politics, Policy and Law, 26(5), 899-911. https://doi. org/10.1215/03616878-26-5-899

Waitzkin, H. y Jasso-Aguilar, R. (2013). Medicina y salud pública al final del imperio. Divulgação em Saúde para Debate, (49), 90-99. 